\title{
Isokinetic Profile of Knee Extensor and Flexor Muscles in Patellofemoral Pain Syndrome Patients
}

\author{
Hasna Ouazzani $^{1 *}$, Davelle Doungou Ngandzali ${ }^{1}$, Youness Abdelfettah ${ }^{1}$
}

\author{
${ }^{1}$ Department of Physical and Rehabilitation Medicine, University Hospital Mohammed VI Marrakesh, Faculty of Medicine, Cadi \\ Ayyad University, Marrakesh, Morocco
}

DOI: $10.36347 /$ sasjm.2021.v07i04.003

| Received: 16.03.2021 | Accepted: 21.04.2021 | Published: 27.04.2021

*Corresponding author: Hasna Ouazzani

Abstract

Original Research Article

Purpose: The aim of the present investigation was to determine the influence of patellofemoral pain syndrome (PFPS) on knee extensors and flexors. Methods: 58 subjects diagnosed with PFPS underwent an isokinetic concentric examination at $60 \% \mathrm{~s}$ and at $180 \%$ s. Data were analyzed through descriptive and correlative statistics with a significance level of 0.05. Results: Patients showed lower extension peak torques compared to body weight and lower muscle peak torques on the involved knee compared to the healthy one, but a normal flexors to extensors ratio. Difference between genders concerned peak torques and total work. Relationships between anthropometric and isokinetic data existed only in females. Pain levels were not correlated to isokinetic measurements. A bigger male sample size and eccentric testing might be necessary to expose other correlations. Conclusion: Our results suggest that strengthening both knee extensors and flexors must be considered in the treatment and prevention of PFPS.

Keywords: Anterior knee pain - concentric - isokinetic - knee - patellofemoral pain syndrome.

Copyright $(\odot 2021$ The Author(s): This is an open-access article distributed under the terms of the Creative Commons Attribution 4.0 International License (CC BY-NC 4.0) which permits unrestricted use, distribution, and reproduction in any medium for non-commercial use provided the original author and source are credited.

\section{INTRODUCTION}

Patellofemoral pain syndrome (PFPS) is a very common cause of anterior knee pain, often encountered in young individuals, due to repetitive flexion stress and poor patellar kinetics. In the absence of degenerative lesions or bad patella centering, the problem is frequently attributed to a strength imbalance between knee flexors and extensors muscles.

Isokinetic testing has become an increasingly popular modality during the last decades and the Cybex HUMAC NORM® isokinetic dynamometer has been widely used in clinical testing to objectively assess factors of muscle performance that would otherwise be difficult to obtain.

It would be interesting to gather knee flexion and extension isokinetic parameters in PPS subjects to create an isokinetic profile that can be used later on as reference for clinical and research purposes.

\section{MATERIALS \& METHODS}

Our study was conducted for a 2 years period, between January 2018 and January 2020.

\section{Participants}

58 subjects took part in this study. The group consisted of 39 women and 19 men, their age ranging from 22 to 68 years with a mean of 35.8 years for women and 34.3 years for men.

Criteria for inclusion were:

- A minimum of three months duration of PFPS with a mild to severe retro or peripatellar pain, especially during walking, jogging, using stairs and/or inclined surfaces, squatting, prolonged sitting with flexed knees ;

- Blockage or giving way episodes ;

- Positive signs of patellofemoral compression: pain on direct compression of the patella, tenderness on palpation of the posterior surface of the patella, pain on resisted knee extension, Zohlen sign.

In addition, negative findings in the examination of knee ligaments, menisci, bursae, synovial plicae, Hoffa's fat pad, iliotibial band, and the hamstrings, quadriceps and patellar tendons and their insertions; plus the absence of other knee pathologies(inflammatory or orthopedic afflictions); 
and of former knee surgery; were also necessary in order to include the subjects in the PFPS study.

All PFPS subjects underwent a detailed physical examination including parameters relating to muscle build, range of motion, limb alignment and patellar orientation. In addition, a series of X-ray and/or CT scans and or MRI were performed. The characteristics of the individuals are presented in Table1 .

Table-1: Anthropometric and clinical characteristics of the PFPS patients

\begin{tabular}{|l|l|l|l|}
\hline Characteristics & Males & Females & p value \\
\hline AGE (years)* & $34.31 \pm 7.86$ & $35.82 \pm 11.9$ & 0.6193 \\
\hline BMI (Kg/m2)* & $25.58 \pm 2.89$ & $26.14 \pm 4.26$ & 0.6092 \\
\hline Pain on VAS* & $3.84 \pm 1.25$ & $4.10 \pm 1.61$ & 0.5407 \\
\hline SIDE OF INJURY & Unilat dom: 6 & Unilat dom: 3 & \\
& Unilat non dom: 7 & Unilat non dom: 8 & \\
& Bilat: 6 & $12.8 \%$ & \\
\hline LOWER EXTREMITY MALALIGNMENT & $10.52 \%$ & $7.68 \%$ & \\
\hline FLAT FEET & $10.52 \%$ & $61.92 \%$ & \\
\hline ZOHLEN TEST + & $47.34 \%$ & $33.28 \%$ & \\
\hline PATELLAR MALPOSITION & $36.82 \%$ & \\
\hline
\end{tabular}

\section{Procedures}

Clinical examination has noted the anthropometric data: weight, height and BMI. Prior to knee joint testing, the subjects undertook a warm-up consisting of 10 to $15 \mathrm{~min}$ on an ergocycle. Then the principle of testing was thoroughly explained, followed up by a familiarization trial on the machine consisting of four submaximal concentric contraction cycles at a submaximal effort. The isokinetic testing was done using the Cybex HUMAC NORM® dynamometer.

Subjects were tested in the sitting position at $85^{\circ}$ hip flexion, with a support for the lower back, a fixation girdle around the thigh, shoulders and abdomen, arms holding at two stabilization bars. The center of motion of the lever arm was aligned as accurately as possible with the flexion-extension axis of the knee joint and the resistance pad was placed at two digits above the external malleolus. The knee was tested from full extension to 120 of flexion. The effect of shank gravity was systematically withdrawn. Following familiarization sets, the actual examination began. The isokinetic evaluation was carried out using the concentric mode. Subjects were instructed to perform repetitive flexion and extension cycles as fast as possible with maximum effort. Protocol comprised a set of 5 repetitions at $60 \% \mathrm{sec}$ angular velocity, a set of 15 repetitions at $180^{\circ} / \mathrm{sec}$, then a last set of 30 repetitions at $180^{\circ} / \mathrm{sec}$ (Table-2). Between each set, a pause of 1 minute was observed. The same protocol was then applied to the other knee. All subjects were encouraged both verbally by the examiner, and visually by following the visual feedback displayed in real time on the computer screen.

An example of parameters recorded in the isokinetic report is presented in Figure-1.

Table-2: Overview of isokinetic testing protocol

\begin{tabular}{|c|c|c|c|}
\hline Speed & Sessions & Mode & Resting Time Between Each Session \\
\hline $60^{\circ} / \mathrm{sec}$ & 5 repetitions & & \\
\hline $180^{\circ} / \mathrm{sec}$ & $\begin{array}{l}15 \text { repetitions } \\
30 \text { repetitions }\end{array}$ & Concentric & 1 minute \\
\hline
\end{tabular}

\section{Data Collection \& Analysis}

Visual analogue scale was used to assess pain that patients experienced during daily activities.

The following variables were studied: extensors' and flexors' peak torques at $60 \%$, and $180^{\circ} \mathrm{s}$, extension peak torque to bodyweight, flexors' to extensors' Ratio, Total work at $180 \%$ s in flexion and extension and Right/Left extension deficit at $60 \%$ s.

The data collected from the Cybex ${ }^{\circledR}$ isokinetic recordings was analyzed using Excel software and STHDA online tool. Descriptive statistics included the mean, median, minimum, maximum, and standard deviation. Quadriceps to Hamstring ratios considered were those at $60 \%$ s. In cases of bilateral dysfunction, the limb with lower ratio was considered.

Force-Range of Motion curves were searched for a "break" produced by torque alteration along a particular ROM segment. A torque curve was judged to be irregular when the tracing was accompanied by one or multiple deflections, as illustrated in Figure 2.

For comparisons between groups, regarding the quantitative variables, was considered the Student $\mathrm{t}$ test for independent samples. For intragroup 
comparisons, regarding the quantitative variables, were considered the Student t-test for paired samples.

Pearson chi-squared test was used to evaluate whether or not a linear dependence exists between anthropometric data and isokinetic parameters.

The statistical significance was set at $\mathrm{p} \leq 0.05$ and the confidence intervals at $95 \%$.

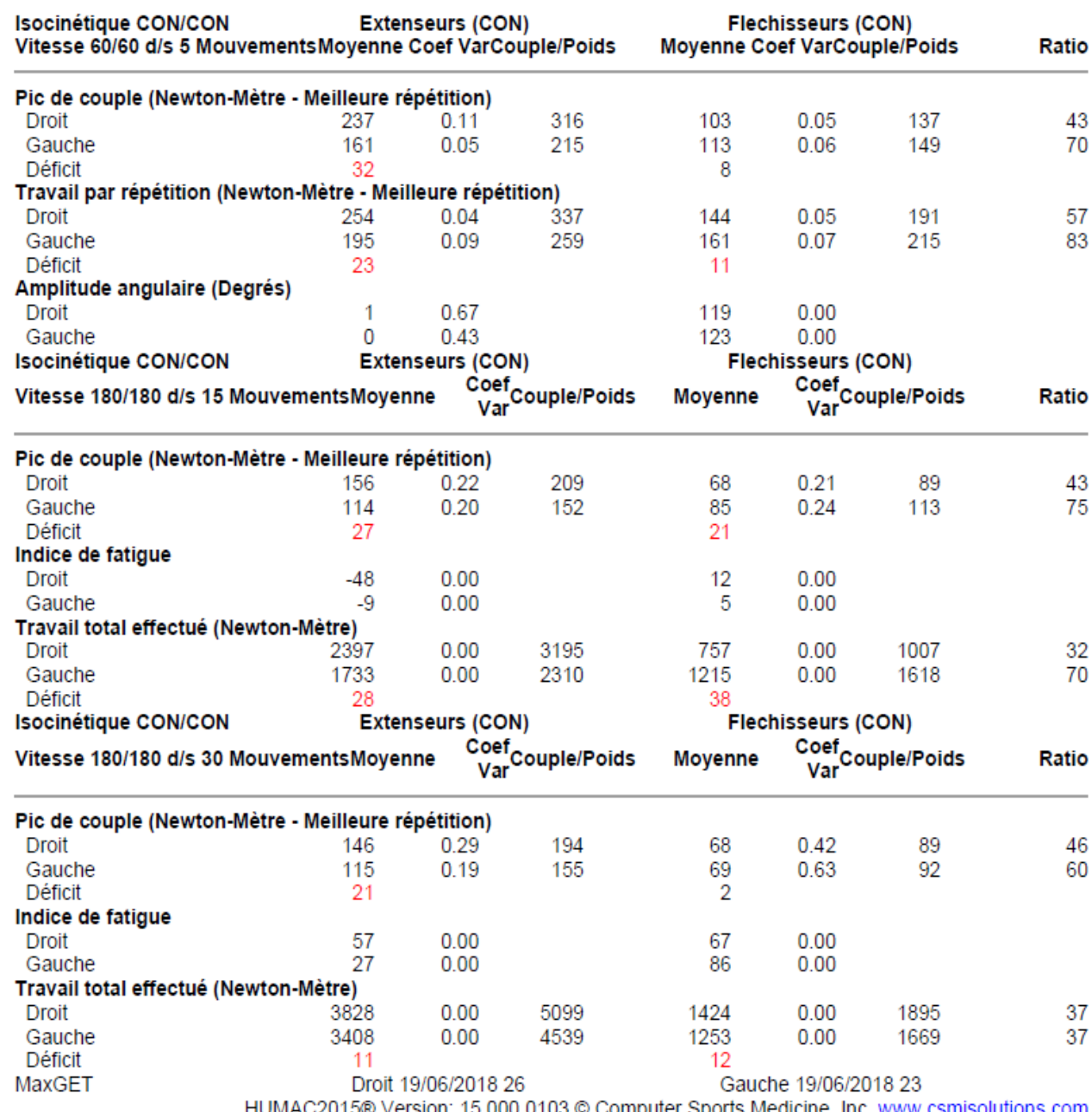

Fig-1: Isokinetic report example
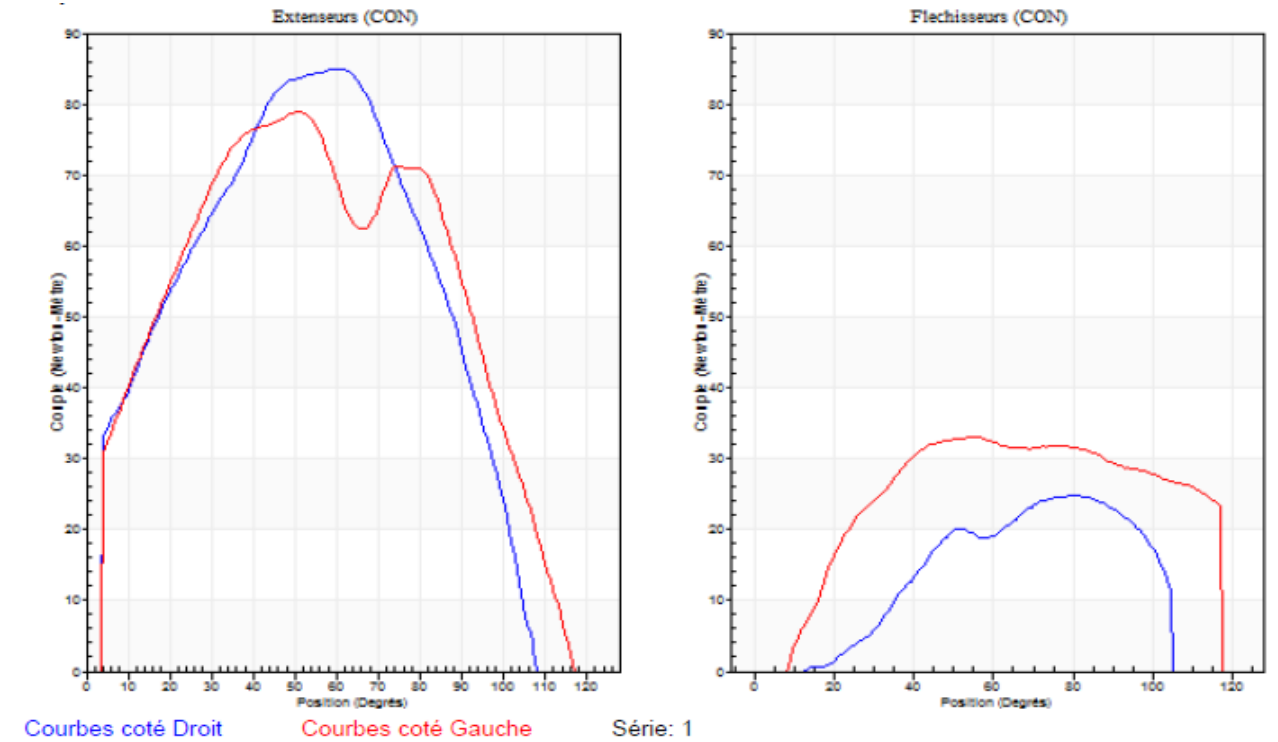

Fig-2: Example of an isokinetic torque curve with a break in the left extensors curve. 
Hasna Ouazzani et al., SAS J Med, Apr, 2021; 7(4): 130-138

\section{RESULTS}

Among participants, there was a female predominance: $67.24 \%$. $58.62 \%$ of patients complained of bilateral symptoms. $43.10 \%$ of subjects had a normal BMI, $32.75 \%$ were overweight, $6.89 \%$ were moderately obese and $3.45 \%$ were severely obese.

$12 \%$ of patients had genu valgum axis deformation. $10.34 \%$ of patients had flat feet. $48.27 \%$ of patients had a $2 \mathrm{~cm}$ circumference difference between the involved thigh and the healthy one. The Zohlen sign was present in $72 \%$ of cases.

$34.48 \%$ of patients had a patellar malposition (alta or tilt) showing on tangential view X-ray imaging. MRI revealed chondromalacia in $6.9 \%$ of patients.

The isokinetic evaluation objectified a lower relative peak extension torque compared to body weight in $87.9 \%$ of patients. There was a significant difference between male's and female's extensors and flexors peak torques at both velocities.

There was also a significant difference between male's and female's total concentric work of extensors and flexors at $180^{\circ} \%$.

The flexors' to extensors' ratio was normal in $72.41 \%$, low in $24.14 \%$ of patients, and high in $3.45 \%$ of patients. The average H/Q ratio was $50.21 \%$ in men and $46.71 \%$ in women. No significant difference was found between male's and female's groups $(p=0.5319)$.

Morphological anomalies in the form of a "break" were present in $44.8 \%$ of cases. $66.67 \%$ of women and $63.15 \%$ of men presented a right/left extensors deficit; while $76.9 \%$ of women $78.9 \%$ of men presented a right/left flexors deficit. Yet, no significant difference was found between genders.

All the data related to isokinetic variables is depicted on Table-3.

Table-3: Isokinetic variables in male and female groups

\begin{tabular}{|c|c|c|c|c|}
\hline & $\begin{array}{l}\text { Male group } \\
\text { MEAN } \pm \text { SD } \\
n=19\end{array}$ & $\begin{array}{l}\text { Female group } \\
\text { MEAN } \pm \text { SD } \\
n=39\end{array}$ & $\begin{array}{l}\text { Total } \\
\text { MEAN } \pm \text { SD } \\
n=58\end{array}$ & P value \\
\hline $\begin{array}{l}\text { Ratio f/e } \\
60^{\circ} / \text { s conc }\end{array}$ & $50.2105 \pm 16.0471$ & $46.7179 \pm 21.4095$ & $48.4642 \pm 18.72845$ & 0.5319 \\
\hline Ext peak torque $60 \% / \mathrm{s}$ conc & $158.3684 \pm 47.3289$ & $89.0513 \pm 22.3747$ & $123.70985 \pm 34.8518$ & $3.385 \mathrm{e}-10$ \\
\hline Flex peak torque $60^{\circ} / \mathrm{s}$ conc & $78.3684 \pm 28.204$ & $46.359 \pm 23.1238$ & $62.3637 \pm 25.6639$ & $2.458 \mathrm{e}-05$ \\
\hline Ext peak torque $180^{\circ} / \mathrm{s}$ conc & $114 \pm 40.2892$ & $55.9487 \pm 14.8571$ & $89.97435 \pm 27.57315$ & $7.528 \mathrm{e}-11$ \\
\hline Flex peak torque $180^{\circ} / \mathrm{s}$ conc & $57.0526 \pm 24.9677$ & $32.1538 \pm 17.8082$ & $44.6032 \pm 21.38795$ & $5.518 e-05$ \\
\hline $\begin{array}{l}\text { Total work } \\
180^{\circ} / \text { s ext conc }\end{array}$ & $1631.1053 \pm 489.2959$ & $847.8462 \pm 285.5915$ & $1239.47575 \pm 387.42525$ & $2.441 \mathrm{e}-10$ \\
\hline $\begin{array}{l}\text { Total work } \\
180^{\circ} / \text { s flex conc }\end{array}$ & $746.8421 \pm 357.6375$ & $378.2051 \pm 233.7431$ & $562.5236 \pm 295.6903$ & $1.662 \mathrm{e}-05$ \\
\hline $\mathrm{R} / \mathrm{L}$ ext deficit $60^{\circ} / \mathrm{s}$ conc & $20.2105 \pm 16.4235$ & $18.0526 \pm 14.4446$ & $19.13155 \pm 15.43405$ & 0.6135 \\
\hline R/L flex deficit $60^{\circ} / \mathrm{s}$ conc & $22.9474 \pm 16.3893$ & $21.2368 \pm 13.1096$ & $22.0921 \pm 14.74945$ & 0.6712 \\
\hline
\end{tabular}

$\mathrm{PT}=$ Peak Torque, conc. $=$ concentric, flex $=$ flexors, ext $=$ extensors, $\mathrm{SD}=$ standard deviation

In patients with unilateral affliction, men made up the majority with a percentage of $54.16 \%$, and the impaired knee was predominantly the non-dominant one $(62.5 \%)$.
In those patients, concentric peak torques of both quadriceps and hamstring muscles were lower on the involved side compared to the uninvolved side at $60 \%$ and at $180 \% \mathrm{~s}$; as was the case for flexors' to extensors' ratios (Table 4). However, no significant difference was observed.

Table-4: Mean peak torques and $\mathrm{F} / \mathrm{E}$ ratios in unilaterally injured patients.

\begin{tabular}{|l|l|l|l|l|}
\hline \multirow{2}{*}{ Speed } & Variable & $\begin{array}{c}\text { Mean } \pm \text { SD } \\
\text { Involved Side }\end{array}$ & Mean \pm SD Uninvolved Side & P Value \\
\hline \multirow{3}{*}{$60^{\circ} / \mathrm{s}$} & Quadriceps PT $(\mathrm{Nm})$ & $121.33 \pm 44.97$ & $129.67 \pm 56.39$ & 0.5741 \\
\cline { 2 - 5 } & Hamstrings PT(Nm) & $58 \pm 27.86$ & $62.46 \pm 26.87$ & 0.5753 \\
\cline { 2 - 5 } & F/E ratio & $47.29 \pm 15.55$ & $48.71 \pm 9.80$ & 0.7075 \\
\hline \multirow{3}{*}{$180^{\circ} / \mathrm{s}$} & Quadriceps PT $(\mathrm{Nm})$ & $82 \pm 31.95$ & $83.42 \pm 31.84$ & 0.8784 \\
\cline { 2 - 5 } & Hamstrings PT(Nm) & $42.5 \pm 22.44$ & $45.04 \pm 23.71$ & 0.7046 \\
\cline { 2 - 5 } & F/E ratio & $48.42 \pm 13.6$ & $51.62 \pm 11.91$ & 0.3891 \\
\hline
\end{tabular}

$\mathrm{PT}=$ Peak Torque, $\mathrm{F}=$ flexors, $\mathrm{E}=$ extensors, $\mathrm{SD}=$ standard deviation 
Hasna Ouazzani et al., SAS J Med, Apr, 2021; 7(4): 130-138

The relationships between different anthropometric variables and isokinetic measurements were examined within the female group and the male group as summarized in Tables 5, 6 and 7 .

Negative correlations were found between extension peak torque and Age on one hand $(r=-$ 0,5781), and extension peak torque and BMI on the other hand $(\mathrm{r}=-0,36)$, but concerned exclusively the group of women.

No correlation was found between level of pain and the isokinetic parameters studied, in neither group.

No correlation was found between Flexors to extensors Ratio $\mathrm{N}$ and the anthropometric nor the isokinetic parameters studied.

Table-5: PEARSON correlations between different anthropometric and isokinetic variables in men

\begin{tabular}{|c|c|c|c|c|c|c|c|c|}
\hline Men & \multicolumn{2}{|l|}{ Age } & \multicolumn{2}{|l|}{ BMI } & \multicolumn{2}{|l|}{ VAS } & \multicolumn{2}{|c|}{ F/E ratio 60} \\
\hline & $\mathrm{r}$ & $\mathrm{p}$ & $\mathrm{r}$ & $\mathrm{p}$ & $\mathrm{r}$ & $\mathrm{p}$ & $\mathrm{r}$ & $\mathrm{p}$ \\
\hline Conc Ext peak torque at $60^{\circ} / \mathrm{s}$ & -0.1574 & 0.5198 & 0.1874 & 0.4425 & 0.002 & 0.9936 & & \\
\hline F/E Ratio at $60^{\circ} / \mathrm{s}$ & -0.2269 & 0.3501 & 0.167 & 0.4943 & 0.2795 & 0.2465 & & \\
\hline Total work of Ext at $180^{\circ} / \mathrm{s}$ & -0.029 & 0.9061 & 0.0751 & 0.76 & 0.2397 & 0.323 & 0.2495 & 0.303 \\
\hline
\end{tabular}

*Data in bold indicate significant correlation $(\mathrm{p}<$ or $=0.05)$.

Table-6: PEARSON correlations between different anthropometric and isokinetic variables in women

\begin{tabular}{|l|l|l|l|l|l|l|l|l|}
\hline Women & Age & BMI & \multicolumn{2}{l|}{ VAS } & \multicolumn{2}{l|}{ F/E ratio 60 } \\
\hline & $\mathrm{r}$ & $\mathrm{p}$ & $\mathrm{r}$ & $\mathrm{p}$ & $\mathrm{r}$ & $\mathrm{p}$ & $\mathrm{r}$ & $\mathrm{p}$ \\
\hline Conc Ext peak torque at $60^{\circ} / \mathrm{s}$ & $\mathbf{- 0 . 5 7 8 1}$ & $\mathbf{0 . 0 0 0 1 1 5 9}$ & $\mathbf{- 0 . 3 6}$ & $\mathbf{0 . 0 2 4 3 8}$ & 0.0267 & 0.8717 & & \\
\hline F/E Ratio at $60^{\circ} \mathrm{s}$ & 0.2341 & 0.1514 & 0.1103 & 0.5037 & -0.0295 & 0.8584 & & \\
\hline Total work of Ext at $180^{\circ} / \mathrm{s}$ & -0.1881 & 0.2516 & -0.0196 & 0.9058 & 0.0493 & 0.7658 & 0.0859 & 0.6029 \\
\hline
\end{tabular}

*Data in bold indicate significant correlation $(\mathrm{p}<$ or $=0.05)$.

Table-7: PEARSON correlations between different anthropometric and isokinetic variables in total

\begin{tabular}{|l|l|l|l|l|l|l|l|l|}
\hline Total & Age & \multicolumn{2}{l|}{ BMI } & \multicolumn{2}{l|}{ VAS } & \multicolumn{2}{l|}{ F/E ratio 60 } \\
\hline & $\mathrm{r}$ & $\mathrm{p}$ & $\mathrm{r}$ & $\mathrm{p}$ & $\mathrm{r}$ & $\mathrm{p}$ & $\mathrm{r}$ & $\mathrm{p}$ \\
\hline Conc Ext peak torque at $60^{\circ} / \mathrm{s}$ & $\mathbf{- 0 . 2 9 3 5}$ & $\mathbf{0 . 0 2 5 3 4}$ & -0.1324 & 0.3218 & -0.0486 & 0.7172 & & \\
\hline F/E Ratio at $60^{\circ} / \mathrm{s}$ & 0.1399 & 0.2948 & 0.1148 & 0.3909 & 0.0302 & 0.8219 & & \\
\hline Total work of Ext at $180^{\circ} / \mathrm{s}$ & -0.1312 & 0.3264 & -0.0403 & 0.7639 & 0.0207 & 0.8776 & 0.155 & 0.2454 \\
\hline
\end{tabular}

*Data in bold indicate significant correlation $(\mathrm{p}<$ or $=0.05)$.

Data was visually represented and analyzed with linear regression curves as presented in Figure 3 and 4. As the cloud of dots can attest, the correlations found are weak.

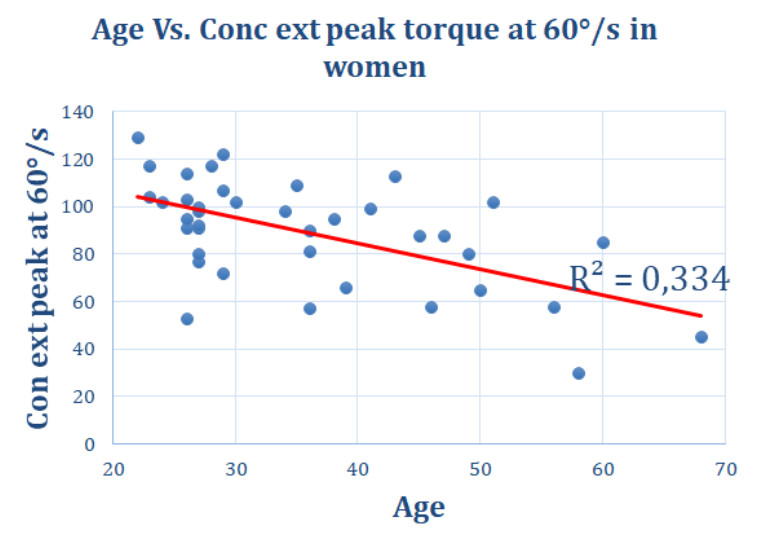

Fig-3: Age Vs. concentric extension peak torque at $60 \%$ in women

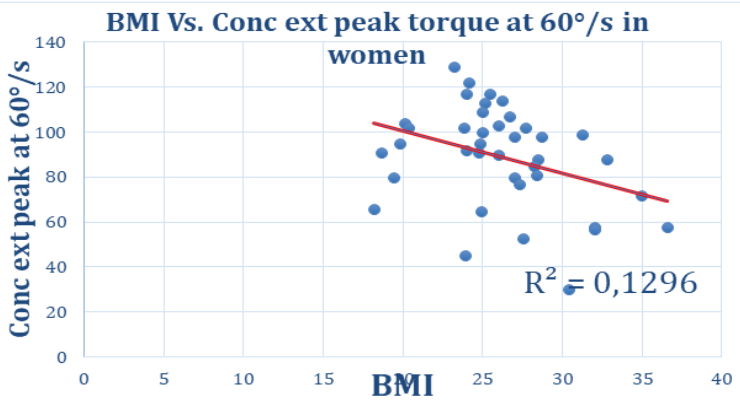

Fig-4: BMI Vs. concentric extension peak torque at $60 \%$ in women

\section{DISCUSSION}

Patellofemoral pain syndrome (PFPS) is a common knee condition characterized by a diffuse anterior knee pain with an insidious onset and a slow progression.

Its incidence is higher in physically active populations, such as teenagers and young adults, especially females, within whom it creates great physical and psychological morbidity by impairing 
Hasna Ouazzani et al., SAS J Med, Apr, 2021; 7(4): 130-138

functional incapacities, thus compromising daily life activities.

The pathophysiology names multiple factors but the most accepted hypothesis is poor patellar alignment [1]. Hypothetically, other factors contribute to the onset or worsening of PFPS, such as chondromalacia, quadriceps weakness, abnormal neuromuscular control of the vastus medialis obliquus and vastus lateralis [2], alterations in the lower extremity postural alignment, especially related to hindfoot and Q angles [3] and abnormalities in the biomechanics of the feet and legs, such as subtalar excessive eversion [4] smaller angle of knee flexion, weak hip muscles [5] in addition to excessive hip adduction and internal rotation [6]. The muscular aspects of PFPS have been the subject of a number of scientific papers.

Isokinetic evaluation is often used to accurately measure muscle function at various modes and speeds, it is then a suitable tool for PFPS analysis.

Many studies have been performed in order to evaluate the torque of knee flexors and extensors using isokinetic dynamometry.

One parameter stood out frequently and has been suggested as a potential cause of PFPS: decreased quadriceps concentric peak torque, which is due to the atrophy or rather the inhibition of quadriceps to prevent pain during knee extension [7]. However, whether decreased torque is, truly, a cause or an effect of PFPS, remains unknown.

In our sample, $87.9 \%$ of patients showed a low peak extension torque. Duffey et al., [8] noted, in a retrospective study, significantly weaker knee extensors peak torque measurements in PFPS patients compared to controls.

A similar result was found in Van Tiggelen et al., study, where absolute and relative peak torques of knee extensors to body weight at $60 \% \mathrm{~s}$ differed significantly between injured and control groups $(\mathrm{p}=0.005)$ [9].

Ott et al., [10] attributed lower knee extensors torque in individuals with PFPS to the inhibition of this muscle because of the pain, especially during functional activities, in the same manner that Powers et al., [11], did by considering this muscle's deficiency a result of strategies adopted to avoid the pain.

On the other hand, Callaghan and Oldham [7] proclaim that lower torque peak in individuals with PFPS cannot be explained by muscle atrophy, considering that they did not find a correlation between the extensors' peak torque and the quadriceps transversal section area, which leads to think that there are more subtle mechanisms, such as modifications in neuromuscular control strategies that eventually limit quadriceps functioning.

It has been documented that men generally have double the muscular strength as that of women. Our study didn't make the exception; indeed, a significant difference was stated between male's and female's both extensors and flexors concentric peak torques at $60 \%$ s as well at $180 \%$ s.

However, many studies advocated that male and female significant variation in muscular strength is related to a culturally enhanced sense of competitiveness in men and higher social pressure to achieve physical prowess [12].

Another important parameter in isokinetic testing is the hamstrings to quadriceps peak torque ratio. Extensors peak torques are documented to be greater than those of the flexors. Though there is no particular ratio that is appropriate for all categories of people, some studies have set a conventional interval between $40 \%$ and $90 \%$ in healthy subjects [12]. That ratio may vary depending on the type of population tested, measuring equipment, test position and angular velocity utilized. But the typical reference landmark is $60 \%$, first suggested by Steindler and then supported by further isokinetic dynamometers investigations making it the optimal proportion for preventing knee injury [13].

This ratio clearly reflects the agonistantagonist knee muscle balance, which means that a weakened quadriceps muscle results in a higher ratio as it is the case in PFPS patients [14].

Our findings didn't quite go in line with those predictions, as $72.41 \%$ of our patients had a normal ratio, $24.14 \%$ had a low one, while only $3.45 \%$ demonstrated a high ratio. This was probably the effect of even lower hamstrings peak torques.

No significant difference was observed when comparing those ratios between gender groups.

The normal shape of the quadriceps torque curve at low velocity isokinetic concentric testing depicts a smooth 'inverted-U'. Variations in this curve associated with malfunctioning muscle-joint systems were described as early as the eighties [15] and are characterized as sudden and considerable reductions in the torque output later termed 'breaks'.

Hart et al., established that the magnitude of these breaks should be greater than the $5 \mathrm{Nm}$ to distinguish them from the small oscillations of the normal curve [16] while Dvir et al., suggested $10 \%$ as the critical value [17]. 
A retrospective study by Hsieh et al., [18] demonstrated that PFPS patients had the highest rate of abnormal torque curves during isokinetic concentric exercise compared to healthy subjects, and occurred at $60 \%$ s, the lowest velocity used in their protocol. However, Elton et al., [19] studied the same type of patients at a high speed $180 \%$ s and didn't find this kind of torque curve abnormalities. In fact, low angular speeds of 30 or $60 \%$ s are believed to increase compressive forces in the patellofemoral joint.

Our subjects, who underwent concentric testing at $60 \% \mathrm{~s}$ and $180 \% \mathrm{~s}$, such breaks on extensor force curves were present in $44.8 \%$ of cases.

The break phenomenon has been attributed to a possible reflex inhibition aimed at joint load release to prevent overstressing the articular facets of the patella by compressive or shear load forces [20].

In Dvir et al., study, breaks occurred exclusively during eccentric isokinetic activity [17].

Lastly, our paper took an interest in examining existing differences between the healthy and pathological knees in the same individual.

The results showed a lower concentric quadriceps and hamstring peak torques on the involved side compared to the uninvolved side at $60 \% \mathrm{~s}$ and $180 \%$ s. But the difference was not significant.

In Kaux et al., study [21], the isokinetic results demonstrated significantly lower quadriceps and hamstrings $60 \%$ seak torques in the pathological limb (respectively $170.5 \pm 44.6$ and $97.2 \pm 21.9$ ) compared to the healthy one (respectively $193.4 \pm 35.6$ and $103.6 \pm$ 22.8).

Besides, there was a significant difference in VAS between the pathological and the contralateral limb in the range of $3.43 \pm 2.80$.

It had been reported that individuals attain peak muscle strength in the third decade of life (between the ages of 20 and 30 years) after which the strength begins to decline gradually so that the strength of a person of 65 years is approximately $80 \%$ of that attained between the ages of 20 and 30 years [22].

This decline in strength with advancing age has been attributed to a reduction in the number of motor neurons and therefore a decline in muscle mass [23].

Our study demonstrated an inverse relationship between quadriceps concentric extension peak torque at $60 \%$ and age $(\mathrm{r}=-0,5781)$ on female patients; this is in agreement with previous reports stating that age affects muscle strength.
One research paper [12] showed that in healthy subjects between 21 and 40 years old, body weight presents a fairly negative correlation with quadriceps muscle strength in male subjects $(-0.643)$ and a poor positive one in male subjects between 41 and 60 years old (0.443). Height was fairly to poorly correlated with quadriceps muscle strength (0.176 to 0.447$)$ depending on age group. The relationship between each of weight and height and the H:Q ratio is generally poor (0.0660.472 ). Correlations were poor to non-existent as regard to female subjects.

Those results are conflicting with ours, mostly as regards to gender implication. In fact, all correlations observed in our case concerned women, only. We can presume that it is a matter of sample size, which was small concerning our male patients.

Moreover, we noted inverse relationships between extensors peak torques and age and BMI. This may be an effect of musculoskeletal and cardiovascular deconditioning resulting from aging and/or a sedentary lifestyle.

At the same time, no correlation was found between H:Q Ratio and anthropometric data, which corresponds well with findings in literature.

The peculiar observation though, was that pain levels were not correlated with isokinetic measurements.

Theoretically, we would have expected quadriceps peak torques to decrease according to increasing levels of pain. The limitation, in our experiment, may be that pain level was determined based on the patient's recalling of pain experienced during daily functional activities. It might have been more suited to evaluate it during actual isokinetic testing.

However, Guney et al., [24] reported significant negative correlations between level of pain during stair ascending and prolonged sitting and concentric quadriceps peak torque on the one hand, and between pain level during stair descending and squatting and prolonged sitting and concentric hamstrings peak torque on the other hand.

In any way, we should bear in mind that pain felt is not necessarily reflective of tissue damage.

One could also argue that our isokinetic examination must have included eccentric testing so as to find a relationship with pain ratings and eccentric outputs, in the manner it had been carried out in Dvir et al., studies [25]. The reason being that eccentric activity produces significantly greater pain than concentric activity. Then again, patellofemoral pain should, ideally, be assessed during functional activities. 


\section{CONCLUSION}

Literature is relatively poor when it comes to a detailed isokinetic PFPS investigation. The main objective of our study was to assess an isokinetic profile in subjects with PFPS by quantifying different parameters and looking for a trend in muscle weakness and muscular unbalance according to age, gender and anthropometric data.

Impairment by PFPS was mainly represented by lower relative peak extension torques compared to body weight, and lower extensors' peak torques in the involved side compared to the healthy side.

Other research protocols including slower velocities, fatigue protocols and eccentric contractions might bring new insight, but these preliminary observations can already help physicians improve PFPS treatment since the examined variables are good indicators of the patient's condition until full recovery.

Conflict of Interest: All authors have no conflicts of interest with respect to the data collected and procedures used within this study. Authors declare that they have no sponsor in the study design, in the collection, analysis and interpretation of data, in writing of the manuscript, and in the decision to submit the manuscript for publication.

\section{Ethical Statement}

The authors confirm that this research paper conforms to the International Committee of Medical Journal Editors (ICMJE) Recommendations for the Conduct, Reporting, Editing, and Publication of Scholarly Work in Medical Journals.

The authors confirm that this research has the approval of the Ethics Committee of the University Hospital Mohammed VI and was conducted according to the Ethical Principles for medical research involving human subjects of the World Medical Association Declaration of Helsinki and that participants provided informed verbal consent to participate in this study.

Funding: The authors report no funding.

\section{REFERENCES}

1. Piazza L, Vidmar MF, Oliveira LF, Pimentel GL, Libardoni TD, Santos GM. Isokinetic evaluation, pain and functionality of subjects with patellofemoral pain syndrome. Fisioterapia e Pesquisa. 2013 Jun;20(2):130-5.

2. Chen HY, Chien CC, Wu SK, Liau JJ, Jan MH. Electromechanical Delay of the Vastus Medialis Obliquus and Vastus Lateralis in Individuals with Patellofemoral Pain Syndrome. Journal of Orthopaedic \& Sports Physical Therapy. 2012 Sep;42(9):791-6.

3. Lankhorst NE, Bierma-Zeinstra SMA, van Middelkoop M. Risk Factors for Patellofemoral
Pain Syndrome: A Systematic Review. Journal of Orthopaedic \& Sports Physical Therapy. 2012 Feb;42(2):81-A12.

4. Levinger P, Gilleard W. Young Investigator Special Issue 1 Research article. 7.

5. Bolgla LA, Malone TR, Umberger BR, Uhl TL. Comparison of hip and knee strength and neuromuscular activity in subjects with and without patellofemoral pain syndrome. International journal of sports physical therapy. $2011 \mathrm{Dec} ; 6(4): 285$.

6. Powers CM. The Influence of Abnormal Hip Mechanics on Knee Injury: A Biomechanical Perspective. Journal of Orthopaedic \& Sports Physical Therapy. 2010 Feb;40(2):42-51.

7. Callaghan MJ. Quadriceps atrophy: to what extent does it exist in patellofemoral pain syndrome? British Journal of Sports Medicine. 2004 Jun 1;38(3):295-9.

8. Duffey MJ, Martin DF, Cannon DW, Craven T, Messier SP. Etiologic factors associated with anterior knee pain in distance runners: Medicine \& Science in Sports \& Exercise. 2000 Nov;32(11):1825-32.

9. Van Tiggelen D, Witvrouw E, Coorevits P, Croisier JL, Roget P. Analysis of isokinetic parameters in the development of anterior knee pain syndrome: A prospective study in a military setting. Isokinetics and Exercise Science. $2004 \mathrm{Dec}$ 28;12(4):223-8.

10. Ott B, Cosby NL, Grindstaff TL, Hart JM. Hip and knee muscle function following aerobic exercise in individuals with patellofemoral pain syndrome. Journal of Electromyography and Kinesiology. 2011 Aug;21(4):631-7.

11. Powers CM, Perry J, Hsu A, Hislop HJ. Are Patellofemoral Pain and Quadriceps Femoris Muscle Torque Associated With Locomotor Function? Physical Therapy. 1997 Oct 1;77(10):1063-75.

12. Jaiyesimi AO, Jegede JS, Adeoluwa OJ. Hamstring and Quadriceps Strength Ratio: Effect Of Age And Gender. Highland Medical Research Journal [Internet]. 2008 Apr 16 [cited 2020 Sep 24];5(1). Available from: http://www.ajol.info/index.php/hmrj/article/view/3 3929

13. Kannus P. Isokinetic Evaluation of Muscular Performance. International Journal of Sports Medicine. 1994 Jan;15(S 1):S11-8.

14. Guney H, Yuksel I, Kaya D, Doral MN. The relationship between quadriceps strength and joint position sense, functional outcome and painful activities in patellofemoral pain syndrome. Knee Surgery, Sports Traumatology, Arthroscopy. 2016 Sep;24(9):2966-72.

15. Hoke B, Howell D, Stack M. The Relationship between Isokinetic Testing and Dynamic Patellofemoral Compression. Journal of Orthopaedic \& Sports Physical Therapy. 1983 Jan;4(3):150-3. 
16. Hart DL, Miller LC, Stauber WT. Effect of cooling on force oscillations during maximal voluntary eccentric exercise. Experimental Neurology. 1985 Oct;90(1):73-80.

17. Dvir Z, Halperin N, Shklar A, Robinson D. Quadriceps Function and Patellofemoral Pain Syndrome. Part II: The Break Phenomenon During Eccentric Activity. Isokinetics and Exercise Science. 1991 Jan 1;1(1):31-5.

18. Hsieh L, Guu C, Liou H, Kung H. Isokinetic and isometric testing of knee musculature in young female patients with patellofemoral pain syndrome. Journal of the Formosan Medical Association. 1992 Feb;91(2):199-205.

19. Elton K, McDonough K, Savinar E, Jensen G. A Preliminary Investigation: History, Physical, and Isokinetic Exam Results Versus Arthroscopic Diagnosis of Chondromalacia Patella. Journal of Orthopaedic \& Sports Physical Therapy. 1985 Nov;7(3):115-23.

20. Lysholm J. The relation between pain and torque in an isokinetic strength test of knee extension. Arthroscopy: The Journal of Arthroscopic \& Related Surgery. 1987 Jan;3(3):182-4.

21. Kaux JF, Croisier JL, Libertiaux V. Isokinetic strength profile of subjects with proximal patellar tendinopathy. Muscle Ligaments and Tendons Journal. 2019 May;9(1):210.

22. Hughes VA, Frontera WR, Wood M, Evans WJ, Dallal GE, Roubenoff R, Singh MA. Longitudinal muscle strength changes in older adults: influence of muscle mass, physical activity, and health. The Journals of Gerontology Series A: Biological Sciences and Medical Sciences. 2001 May 1;56(5):B209-17.

23. Berger MJ, Doherty TJ. Sarcopenia: Prevalence, Mechanisms, and Functional Consequences. In: Mobbs CV, Hof PR, editors. Interdisciplinary Topics in Gerontology [Internet]. Basel: KARGER; 2010 [cited 2020 Sep 24]. p. 94-114. Available from:

https://www.karger.com/Article/FullText/319997

24. Guney H, Yuksel I, Kaya D, Doral MN. Correlation between quadriceps to hamstring ratio and functional outcomes in patellofemoral pain. The Knee. 2016 Aug;23(4):610-5.

25. Dvir Z, Halperin N, Shklar A, Robinson D. Quadriceps Function and Patellofemoral Pain Syndrome. Part I: Pain Provocation During Concentric and Eccentric Isokinetic Activity. Isokinetics and Exercise Science. 1991 Jan 1;1(1):26-30. 